\title{
Stochastic stability of the Ekman spiral
}

\author{
Matthias Hieber AND Wilhelm StanNat
}

\begin{abstract}
Consider the stochastic Navier-Stokes-Coriolis equations in $\mathbb{T}^{2} \times$ $(0, b)$ subject to Dirichlet boundary conditions as well as the Ekman spiral which is a stationary solution to the deterministic equations. It is proved that the stochastic Navier-Stokes-Coriolis equation admits a weak martingale solution. Moreover, as an stochastic analogue of the existing deterministic stability results for the Ekman spiral, stochastic stability of the Ekman spiral is proved by considering stationary martingale solutions.
\end{abstract}

Mathematics Subject Classification (2010): 35R60 (primary); 35Q30, 37L40, 60H15, 76D05, 76M35 (secondary).

\section{Introduction}

Consider the Navier-Stokes equations in the rotational setting on an infinite layer $\mathbb{R}^{2} \times(0, b)=: \Omega$ for some $b>0$,

$$
\left\{\begin{array}{rlrl}
\partial_{t} u-v \Delta u+\omega\left(e_{3} \times u\right)+(u \cdot \nabla) u+\nabla p & =0, & & t>0, x \in \Omega, \\
\operatorname{div} u & =0, & & t>0, x \in \Omega, \\
u\left(t, x_{1}, x_{2}, 0\right) & =0, & & t>0, x_{1} \in \mathbb{R}, x_{2} \in \mathbb{R}, \\
u\left(t, x_{1}, x_{2}, b\right) & =e_{1} \cdot u_{b}, & t>0, x_{1} \in \mathbb{R}, x_{2} \in \mathbb{R}, \\
u(0, \cdot) & =u_{0}, & & x \in \Omega .
\end{array}\right.
$$

Here $\omega \in \mathbb{R}$ denotes the speed of rotation, $v>0$ the viscosity of the fluid, $u_{b} \in \mathbb{R}$ is a constant, and $e_{i}, i=1,2,3$, denotes the $i$-th unit vector in $\mathbb{R}^{3}$. Equations (1.1) are sometimes also called Navier-Stokes-Coriolis equations because of the additional Coriolis forcing term. It is a remarkable fact that (1.1) admits an explicit stationary solution $\left(u_{b}^{E}, p_{b}^{E}\right)$ given by

$$
u_{b}^{E}\left(x_{1}, x_{2}, x_{3}\right)=\tilde{u}_{b}\left(\begin{array}{c}
1-e^{-\frac{x_{3}}{\delta}} \cos \left(\frac{x_{3}}{\delta}\right) \\
e^{-\frac{x_{3}}{\delta}} \sin \left(\frac{x_{3}}{\delta}\right) \\
0
\end{array}\right), p_{b}^{E}\left(x_{1}, x_{2}, x_{3}\right)=-\omega \tilde{u}_{b} x_{2}
$$

Received August 3, 2010; accepted in revised form June 21, 2011. 
with $\delta=\frac{b}{k \pi}, k \in \mathbb{Z}$, and

$$
\tilde{u}_{b}:=u_{b}\left(1-e^{-\frac{b}{\delta}}\right)^{-1} \text { if } k \text { is even and } \tilde{u}_{b}:=u_{b}\left(1+e^{-\frac{b}{\delta}}\right)^{-1} \text { if } k \text { is odd. }
$$

This stationary solution of equation (1.1) is called in honour of the swedish oceanograph V. W. Ekman, the Ekman spiral; see [9]. It describes rotating boundary layers between a geostrophic flow and a solid boundary subject to Dirichlet boundary conditions. Starting from this particular stationary solution, it is a natural problem to investigate its stability properties.

Deterministic perturbations of the Ekman spiral by functions $u$ solving the above equation (1.1) have been considered by many authors. In fact, set

$$
w:=u-u_{b}^{E}, \quad \text { and } \quad q:=p-p_{b}^{E} .
$$

Then the pair $(w, q)$ formally satisfies the equations

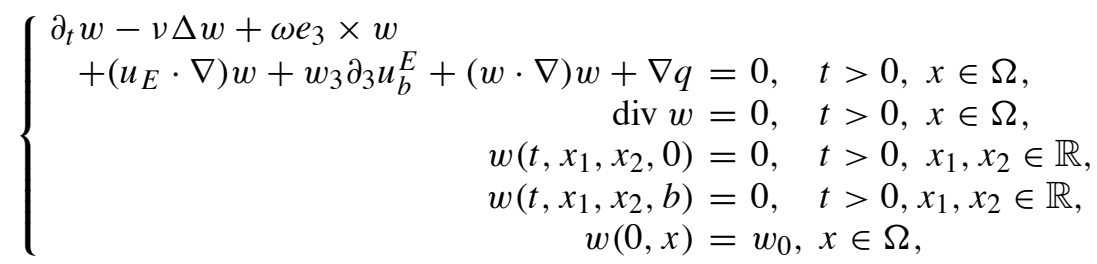

where $w_{0}=u_{0}-u_{b}^{E}$. It was shown by Desjardin, Dormy and Grenier in [8] that the Ekman spiral is stable in the sense that if for all initial data $w_{0} \in L_{\sigma}^{2}(D)$ there exists a global weak solution $w$ to (1.2), then

$$
\|w(t)\|_{2} \leq\left\|w_{0}\right\|_{2} \quad \text { for all } t>0,
$$

holds true provided the Reynolds number $R e$ is small enough. Furthermore, it was shown by Hess in [16] that for every initial data $w_{0} \in L_{\sigma}^{2}(D)$ there exists a global weak solution to (1.2) and that this solution satisfies

$$
\lim _{t \rightarrow \infty}\|w(t)\|_{2}=0
$$

with an exponential rate. Note that this notion of stability corresponds to the definition of asymptotical stability in dynamical systems. It was proved moreover in [17] that the Ekman spiral in the half space $\mathbb{R}_{+}^{3}$ is nonlinearly stable with respect to $L^{2}-$ perturbations provided the corresponding Reynolds number is small enough. Moreover, the decay rate could be computed in terms of the decay of the corresponding linear problem.

For further stability results concerning the Navier-Stokes-Coriolis equations defined on all of $\mathbb{R}^{3}$ we refer to [14] and [18].

Observe that the above stability results concern the situation of a fixed speed of rotation $\omega$, whereas e.g. Rousset's result [23] concerns the situation, where $\omega$ tends 
to $\infty$. Very recently, Gallay and Roussier-Michon considered in [12] the NavierStokes-Coriolis equations in an infinite layer with periodic boundary conditions in the vertical directions. They proved the existence and uniqueness of a global mild solution to the above system for arbitrarily large data belonging to a certain function space, provided $\omega$ is large enough, and investigated its long-time behavior. For related results see also [20] and [3].

The aim of this paper is twofold: first we introduce a stochastic analogue of (1.1) in domains $D$ of the form $D:=\mathbb{T}^{2} \times(0, b)$ by adding an exterior additive stochastic forcing term to (1.1) using a function-space valued Wiener process. Secondly, as the stochastic analogue of the above deterministic stability results we study the stochastic stability of the associated stochastic partial differential equation by considering stationary martingale solutions.

More precisely, we consider in the following the stochastic Navier-StokesCoriolis equations in the space $L^{2, \operatorname{per}}(D)^{3}$, by restricting the problem to $\left(x_{1}, x_{2}\right)$ periodic solutions of (1.1). Indeed, note that since the Ekman spiral is independent of the variables $\left(x_{1}, x_{2}\right)$, it can be viewed as a $\left(x_{1}, x_{2}\right)$-periodic function belonging to $L^{2, \operatorname{per}}(D)$.

The stochastic Navier-Stokes equations in the rotational setting can be formulated as

$$
\left\{\begin{aligned}
d u_{t} & =\left[v \Delta u_{t}-\omega\left(e_{3} \times u_{t}\right)-\left(u_{t} \cdot \nabla\right) u_{t}+\nabla p_{t}\right] d t+d W_{t} \\
\operatorname{div} u_{t} & =0 \\
u_{t}\left(x_{1}, x_{2}, 0\right) & =0 \\
u_{t}\left(x_{1}, x_{2}, b\right) & =e_{1} \cdot u_{b}
\end{aligned}\right.
$$

where $\left(W_{t}\right)$ is a Wiener process on the subspace $H \subset L^{2, p e r}(D)^{3}$ of the closure of smooth $\left(x_{1}, x_{2}\right)$-periodic vector-fields having divergence zero. We will reformulate (1.4) as a stochastic evolution equation and consider the resulting stochastic partial differential equation for the velocity

$$
d u_{t}=\left[v A_{S} u_{t}-\omega \Pi\left(e_{3} \times u_{t}\right)-\Pi\left(u_{t} \cdot \nabla u_{t}\right)\right] d t+d W_{t}
$$

on the space $H$. Here $\Pi: L^{2, \operatorname{per}}(D)^{3} \rightarrow H$ denotes the Helmholtz projection and $A_{S}=\Pi \Delta$ the Stokes operator in $H$. For a precise definition of $\Pi$ and the Stokes operator in this context, we refer to Section 2. Equation (1.5) can be regarded as a stochastic Navier-Stokes-Coriolis equation.

Stochastic Navier-Stokes equations are studied already for quite some time; we refer to the pioneering works of Bensoussan and Temam [4] and Vishik and Fursikov [26] for existence of weak solutions. Of particular interest to us is the seminal paper [10] by Flandoli and Gatarek concerning the existence of (stationary) martingale solutions of stochastic Navier-Stokes equations.

Similarly to the deterministic case, for a solution $u_{t}$ of (1.5), we consider

$$
u_{t}=v_{t}+u_{b}^{E}
$$


where $u_{b}^{E}$ denotes the Ekman spiral. Then $v_{t}$ is a solution of the semilinear stochastic evolution equation

$$
d v_{t}=\left[\left(\nu A_{S}+B\right) v_{t}-\Pi\left(v_{t} \cdot \nabla v_{t}\right)\right] d t+d W_{t}
$$

where $B$ is defined by

$$
B v:=-\omega \Pi\left(e_{3} \times v\right)-\Pi\left(u_{b}^{E} \cdot \nabla v\right)-\Pi\left(v_{3} \partial_{3} u_{b}^{E}\right), \quad v \in D\left(A_{S}\right) .
$$

In Section 3 we present the main result of this paper concerning existence of weak martingale solutions of (1.6). Our proof is very much inspired by the proof of Theorem 3.2 in [10], the main differences to [10] are due to the additional Ekman and Coriolis terms and the different boundary conditions for the Stokes operator. For simplicity we restrict ourselves to the case of Wiener-processes $\left(W_{t}\right)$ having finite trace covariance $Q$.

As a stochastic analogue of the deterministic stability of the Ekman spiral, we study in Section 4 the stochastic stability of (1.5) by considering stationary martingale solutions. In particular, the time-invariant distribution $\mu$ of a stationary martingale solution can be interpreted as the long-time statistics of random fluctuations of (1.5) around the Ekman spiral. In Corollary 4.3 we prove the existence of stationary martingale solutions with invariant distribution $\mu$ satisfying the exponential moment estimate

$$
\int e^{\varepsilon\|u\|_{H}^{2}} d \mu(u)<\infty
$$

for small $\varepsilon$. This means that the fluctuations of (1.5) around the Ekman spiral have exponential tails.

Similar moment estimates for stochastic Navier-Stokes equations in the classical setting, i.e. without rotation, can be found e.g. in [5,24] and [22].

\section{Deterministic Navier-Stokes-Coriolis equations}

Let $D:=\mathbb{T}^{2} \times(0, b)$ for some $b>0$. We first introduce Sobolev spaces consisting of functions being periodic in $\left(x_{1}, x_{2}\right)$. Given a fixed number $l>0$, let $\mathcal{D}$ be the space of all functions $f: \bar{D} \rightarrow \mathbb{C}$ which can be expressed as

$$
f\left(x^{\prime}, x_{3}\right)=\sum_{k \in J} \hat{f}_{k}\left(x_{3}\right) e^{i z<k, x^{\prime}>}
$$

for some finite subset $J$ of $\mathbb{Z}^{2}$ and some $\hat{f}_{k} \in C^{\infty}([0, b])$, where $z=\frac{\pi}{l}$. Then $\left\{e^{i z<k, \cdot>}: k \in \mathbb{Z}\right\}$ is an orthogonal set in $L^{2}(T)$ with $T=[-l, l]^{2}$. Note that the coefficients $\hat{f}_{k}$ of $f$ are uniquely determined by the partial Fourier series of $f$ by

$$
\hat{f}_{k}\left(x_{3}\right)=\frac{1}{(2 \pi)^{2}} \int_{T} f\left(x^{\prime}, x_{3}\right) e^{-i z<k, x^{\prime}>} d x^{\prime}, \quad k \in \mathbb{Z}^{2} .
$$


We define $\mathcal{D}_{0, \sigma}$, the space of divergence free test functions, as

$$
\mathcal{D}_{0, \sigma}:=\left\{f \in[\mathcal{D}]^{3}: \operatorname{div} f=0 \text { in } D \text { and } f=0 \text { on } \partial D\right\} .
$$

Given $m \in \mathbb{N}_{0}$ and $f \in D$, we set

$$
\|f\|_{2}^{2}=\int_{0}^{b} \int_{T}\left|f\left(x^{\prime}, x_{3}\right)\right|^{2} d x^{\prime} d x_{3} \text { and }\|f\|_{m, 2}^{2}=\sum_{|\alpha| \leq m}\left\|D^{\alpha} f\right\|_{2}^{2} .
$$

Then our basic function spaces are defined as the closures of $\mathcal{D}$ or $\mathcal{D}_{0, \sigma}$ with respect to $\|\cdot\|_{m, 2}$ as

$$
H^{m, 2, \text { per }}={\overline{[\mathcal{D}]^{3}}}^{\|\cdot\|_{m, 2}}, \quad L_{\sigma}^{2, \text { per }}={\overline{\mathcal{D}_{0, \sigma}}}^{\|\cdot\|_{2}}, \quad H_{0, \sigma}^{1,2, \text { per }}={\overline{\mathcal{D}_{0, \sigma}}}^{\|\cdot \cdot\|_{1,2}} .
$$

We next introduce the Helmholtz projection from $L^{2, \operatorname{per}}(D)$ onto $L_{\sigma}^{2, \text { per }}(D)$. Note first that the existence of the Helmholtz projection for spaces of the form $L^{2}(D)$ is well known; see e.g. [25] or [11]. Adapting their arguments to partial Fourier series one obtains the following result. For each $u \in L^{2}$, $\operatorname{per}_{(D)}$, there exists a unique $v \in L_{\sigma}^{2, p e r}(D)$ satisfying $u=v+\nabla p$ for some scalar $p \in H^{1,2, \operatorname{per}_{(}}(D)$ and a constant $C>0$ such that

$$
\|v\|_{2}+\|\nabla p\|_{2} \leq C\|u\|_{2}
$$

Hence, the mapping

$$
u \in L^{2, \operatorname{per}_{(}}(D) \mapsto v=\Pi u \in L_{\sigma}^{2, \operatorname{per}}(D)
$$

defines a bounded linear operator $\Pi$ from $L^{2, \operatorname{per}_{(}}(D)$ onto $L_{\sigma}^{2, \text { per }}(D)$, which is called the Helmholtz projection. The Stokes operator $A_{S}$ in $L_{\sigma}^{2, \text { per }}(D)$ is then defined by

$$
A_{S} u:=\Pi \Delta u \quad \text { with } \quad D\left(A_{S}\right):=H_{0, \sigma}^{1,2, \operatorname{per}}(D) \cap H^{2,2, \operatorname{per}_{(}}(D) .
$$

Note that the Stokes operator $A_{S}$ could be defined alternatively also by variational methods, see e.g. [25]. Observe, however, that then the above characterization of the domain $D\left(A_{S}\right)$ for mixed periodic-Dirichlet boundary data is not obvious.

Adapting the arguments given in [1,2] or [15] to the mixed periodic-Dirichlet setting, we see that the Stokes operator has the following spectral properties.

Lemma 2.1. Let $0<\varepsilon<\frac{\pi}{2}$ and $\lambda \in \Sigma_{\pi-\varepsilon}:=\{z \in \mathbb{C} \backslash\{0\}:|\arg z|<\pi-\varepsilon\}$. Then for $f \in L_{\sigma}^{2, p e r}(D)$ there exists a unique $u \in D\left(A_{S}\right)$ satisfying $\left(\lambda-v A_{S}\right) u=f$ and a constant $C=C(\varepsilon)>0$ such that

$$
|\lambda|\|u\|_{2}+v\|u\|_{2,2} \leq C\|f\|_{2} .
$$


The above lemma implies in particular that the Stokes operator $A_{S}$ generates an analytic semigroup on $L_{\sigma}^{2, p e r}(D)$ and that the set $\mathbb{C} \backslash(-\infty, 0]$ is contained in the resolvent set of $A_{S}$. Moreover, since the estimate (2.1) is uniform in $\lambda \in(0, \infty)$, it follows that

$$
0 \in \varrho\left(A_{S}\right) \quad \text { and } \quad \nu\left\|\left(A_{S}\right)^{-1} f\right\|_{2,2} \leq C\|f\|_{2}
$$

for some constant $C>0$. A more detailed analysis even shows that $\mathbb{C} \backslash\left(-\infty,-\frac{\pi^{2}}{b^{2}}\right] \subset$ $\varrho\left(A_{S}\right)$.

The precise description of $D\left(A_{S}\right)$ will be important in step 3 of the proof of our main result, where the tightness of the sequence $P^{n}$ of projection on the span of the first $n$ eigenvectors of the Stokes operator is proved by the compactness of certain embeddings.

Finally, for $V:=H_{0, \sigma}^{1,2, p e r}(D)$, equipped with the equivalent norm $\|u\|_{V}^{2}:=$ $\left\langle-A_{S} u, u\right\rangle_{H}$, consider operators $B: V \rightarrow H$ for which there exist constants $\omega_{0}>$ $0, \omega_{1} \geq 0$ satisfying

$$
\left\langle\left(v A_{S}+B\right) u, u\right\rangle_{H} \leq-\omega_{0}\|u\|_{V}^{2}+\omega_{1}\|u\|_{H}^{2}, \quad u \in D\left(A_{S}\right) .
$$

It then follows by standard perturbation theory that $\nu A_{S}+B$ with domain $D\left(A_{S}\right)$ generates an analytic semigroup on $H$.

\section{Stochastic Navier-Stokes-Coriolis equations}

We start this section by adding noise as an independent exterior forcing term to (1.1) and obtain the equation

$$
\left\{\begin{aligned}
d u_{t} & =\left[v \Delta u_{t}-\omega\left(e_{3} \times u_{t}\right)-\left(u_{t} \cdot \nabla\right) u_{t}+\nabla p_{t}\right] d t+d W_{t} \\
\operatorname{div} u_{t} & =0 \\
u_{t}\left(x_{1}, x_{2}, 0\right) & =0 \\
u_{t}\left(x_{1}, x_{2}, b\right) & =e_{1} \cdot u_{b} .
\end{aligned}\right.
$$

Here, $\left(W_{t}\right)_{t \geq 0}$ is an $H$-valued $Q$-Wiener process, where $H:=L_{\sigma}^{2, \text { per }}(D)$, defined on an underlying stochastic basis $\left(\Omega, \mathcal{F},\left(\mathcal{F}_{t}\right)_{t \geq 0}, P\right)$ (see [6, Chapter 4]). Note that the covariance operator $Q$ of $\left(W_{t}\right)_{t \geq 0}$ necessarily has finite trace.

Applying the Helmholtz projection $\Pi$ introduced in Section 2 to (3.1) leads to the following semilinear stochastic evolution equation

$$
d u_{t}=\left[v A_{S} u_{t}-\omega \Pi\left(e_{3} \times u_{t}\right)-\Pi\left(u_{t} \cdot \nabla u_{t}\right)\right] d t+d W_{t}
$$

in $H$. Let $V^{\prime}=H_{0, \sigma}^{-1,2, p e r}(D)$ be the (topological) dual space of $V$. Identifying $H$ with its dual $H^{\prime}$ we have that $V \hookrightarrow H \equiv H^{\prime} \hookrightarrow V^{\prime}$ densely and continuously. 
We denote by $V^{\prime}\langle u, v\rangle_{V}, u \in V^{\prime}, v \in V$, the dualization of $V^{\prime}$ with $V$. Note that $V^{\prime}\langle u, v\rangle_{V}=\langle u, v\rangle_{H}$ if $u \in H$.

As described above, for a solution $u_{t}$ of (1.5), we consider

$$
u_{t}=v_{t}+u_{b}^{E},
$$

where $u_{b}^{E}$ denotes the Ekman spiral. Then $v_{t}$ is a solution of the semilinear stochastic evolution equation

$$
d v_{t}=\left[\left(v A_{S}+B\right) v_{t}-\Pi\left(v_{t} \cdot \nabla v_{t}\right)\right] d t+d W_{t}
$$

where $B$ is defined by

$$
B v:=-\omega \Pi\left(e_{3} \times v\right)-\Pi\left(u_{b}^{E} \cdot \nabla v\right)-\Pi\left(v_{3} \partial_{3} u_{b}^{E}\right), \quad v \in D\left(A_{S}\right) .
$$

We will study in the following the equation (1.6) not only for $B$ defined as above, but for linear operators $B$ satisfying the following two assumptions:

(A1) $B: V \rightarrow H$ is a bounded linear operator such that there exist $\omega_{0}>0, \omega_{1} \geq 0$ with

$$
\left\langle\left(v A_{S}+B\right) u, u\right\rangle_{H} \leq-\omega_{0}\|u\|_{V}^{2}+\omega_{1}\|u\|_{H}^{2}, \quad u \in D\left(A_{S}\right) .
$$

(A2) The covariance operator $Q$ of $(W(t))_{t \geq 0}$ has finite trace.

Remarks 3.1. a) We note that (A2) implies that there exists an orthonormal basis $\left(f_{k}\right)_{k \geq 1}$ of $H$ consisting of eigenvectors of $Q$ with corresponding eigenvalues $\left(\mu_{k}^{2}\right)_{k \geq 1}$ such that $\left(W_{t}\right)_{t \geq 0}$ can be represented as

$$
W_{t}=\sum_{k=1}^{\infty} \mu_{k} f_{k} \cdot \beta_{k}(t),
$$

where $\left(\beta_{k}(t)\right)_{t \geq 0}, k \geq 1$, are independent, 1-dimensional Brownian motions on $(\Omega, \mathcal{F}, P)$.

b) Note also that (A1) implies as described in Section 2 that the operator $v A_{S}+B$ with domain $D\left(A_{S}\right)$ generates an analytic $C_{0}$-semigroup $\left(T_{t}\right)_{t \geq 0}$ on $H$ satisfying $\|T(t)\| \leq e^{\left(\omega_{1}-\frac{\pi^{2}}{b^{2}} \omega_{0}\right) t}$ for all $t \geq 0$.

We now introduce the notion of a martingale solution to equation (1.6).

Definition 3.2. A martingale solution of (1.6) is a pair $\left(\left(v_{t}\right)_{t \geq 0},\left(\tilde{W}_{t}\right)_{t \geq 0}\right)$, defined on a stochastic basis $\left(\tilde{\Omega}, \tilde{\mathcal{F}},\left(\tilde{\mathcal{F}}_{t}\right)_{t \geq 0}, \tilde{P}\right)$ satisfying

(i) $\left(\tilde{W}_{t}\right)_{t \geq 0}$ is an $\left(\tilde{\mathcal{F}}_{t}\right)_{t \geq 0}$ Wiener-process with covariance $Q$,

(ii) $v .:[0, T] \times \tilde{\Omega} \rightarrow H$ is progressively measurable,

(iii) $v . \in L^{\infty}([0, T] ; H) \cap L^{2}([0, T] ; V) \quad \tilde{P}-$ a.s., 
(iv) For all $u \in V$ and all $t \in[0, T] \tilde{P}-$ a.s.

$$
\begin{aligned}
\left\langle v_{t}, u\right\rangle_{H}= & \left\langle v_{0}, u\right\rangle_{H}+\int_{0}^{t} V^{\prime}\left\langle\left(v A_{S}+B\right) v_{s}, u\right\rangle_{V} d s \\
& -\int_{0}^{t}\left\langle\Pi\left(v_{s} \cdot \nabla v_{s}\right), u\right\rangle_{H} d s+\left\langle\tilde{W}_{t}, u\right\rangle_{H} .
\end{aligned}
$$

The following theorem is the main result of this section.

Theorem 3.3. Assume (A1) and (A2). Let $\xi: \Omega \rightarrow H$ be $\mathcal{F}_{0}$-measurable, squareintegrable and independent of $\left(W_{t}\right)_{t \geq 0}$. Then for all $T>0$ there exists a martingale solution $\left(\left(v_{t}\right)_{t \geq 0},\left(\tilde{W}_{t}\right)_{t \geq 0}\right)$ of (1.6) satisfying $\tilde{P} \circ v_{0}^{-1}=P \circ \xi^{-1}$. Moreover,

$$
\tilde{E}\left(\sup _{0 \leq t \leq T}\left\|v_{t}\right\|_{H}^{2}+\int_{0}^{T}\left\|v_{t}\right\|_{V}^{2} d t\right)<\infty .
$$

The following proof of Theorem 3.3 follows closely the proof of [10, Theorem 3.2]. To this end, denote by $\left(e_{k}\right)_{k \geq 1}$ an orthonormal basis of $H$ consisting of eigenvectors of $A_{S}$.

\section{Step 1: Galerkin approximations}

Denote by

$$
P_{n}: H \rightarrow \operatorname{span}\left\{e_{k}: k \leq n\right\} \subset H, x \mapsto \sum_{k=1}^{n}\left\langle x, e_{k}\right\rangle e_{k}
$$

the orthogonal projection onto the linear subspace of $H$ given as the linear span of the first $n$ eigenvectors and consider for fixed $n$ the following stochastic evolution equation:

$$
\begin{aligned}
d v_{t}^{n} & =\left[P_{n}\left(v A_{S}+B\right) v_{t}^{n}+P_{n} F_{n}\left(v_{t}^{n}, v_{t}^{n}\right)\right] d t+d P_{n} W_{t}, \\
v_{0}^{n} & =P_{n} \xi_{0}
\end{aligned}
$$

Here,

$$
F_{n}(u, v):=\Theta_{n}\left(\|u\|_{H}\right)(u \cdot \nabla) v, \quad u, v \in P_{n} H,
$$

for some continuously differentiable cutoff-function $\Theta_{n}: \mathbb{R} \rightarrow \mathbb{R}$ satisfying

$$
1_{[-n, n]} \leq \Theta_{n} \leq 1_{[-n-1, n+1]} .
$$

Note that for $u, v \in P_{n} H$ we have

$$
\left\langle P_{n} F_{n}(u, v), v\right\rangle_{H}=\left\langle F_{n}(u, v), v\right\rangle_{H}=\left\langle\Theta_{n}\left(\|u\|_{H}\right)(u \cdot \nabla) v, v\right\rangle_{H}=0 .
$$

The above equation (3.5) can be viewed as a finite dimensional stochastic differential equation having locally Lipschitz continuous coefficients of at most linear 
growth. Now [21, Theorem 1 in Section V.1] implies that (3.5) has a unique strong solution satisfying in addition the following moment estimate

$$
E\left(\sup _{0 \leq t \leq T}\left\|v_{t}^{n}\right\|_{H}^{2}\right)<\infty
$$

\section{Step 2: Energy estimate}

In this step we will derive the uniform energy estimate

$$
E\left(\sup _{0 \leq t \leq T}\left\|v_{t}^{n}\right\|_{H}^{2}+\int_{0}^{T}\left\|v_{t}^{n}\right\|_{V}^{2} d t\right) \leq M<\infty
$$

for some finite constant $M$ independent of $n$. In order to establish (3.7), note that by Ito's formula

$$
\begin{aligned}
\frac{1}{2} e^{-\omega_{1} t}\left\|v_{t}^{n}\right\|_{H}^{2}= & \frac{1}{2}\left\|P_{n} \xi_{0}\right\|_{H}^{2}+\int_{0}^{t} e^{-\omega_{1} s}\left\langle v_{s}^{n}, d P_{n} W_{s}\right\rangle_{H} \\
& +\int_{0}^{t} e^{-\omega_{1} s}\left(\left\langle v_{s}^{n}, P_{n}\left(v A_{s}+B\right) v_{s}^{n}+P_{n} F_{n}\left(v_{s}^{n}, v_{s}^{n}\right)\right\rangle_{H}-\omega_{1}\left\|v_{s}^{n}\right\|_{H}^{2}\right) d s \\
& +\frac{1}{2 \omega_{1}}\left(1-e^{-\omega_{1} t}\right) \operatorname{tr}_{H}\left(P_{n} \circ Q\right) .
\end{aligned}
$$

Taking into account (3.6) and assumption (A1) we obtain

$$
\begin{aligned}
\left\langle v_{s}^{n}, P_{n}\left(v A_{S}+B\right) v_{s}^{n}+P_{n} F_{n}\left(v_{s}^{n}, v_{s}^{n}\right)\right\rangle_{H} & =\left\langle v_{s}^{n},\left(v A_{s}+B\right) v_{s}^{n}\right\rangle_{H} \\
& \leq-\omega_{0}\left\|v_{s}^{n}\right\|_{V}^{2}+\omega_{1}\left\|v_{s}^{n}\right\|_{H}^{2} .
\end{aligned}
$$

Inserting the last inequality into (3.8) and rearranging terms we obtain

$$
\begin{aligned}
& \frac{1}{2} e^{-\omega_{1} t}\left\|v_{t}^{n}\right\|_{H}^{2}+\omega_{0} \int_{0}^{t} e^{-\omega_{1} s}\left\|v_{s}^{n}\right\|_{V}^{2} d s \\
& \leq \frac{1}{2}\left\|P_{n} \xi_{0}\right\|_{H}^{2}+\frac{1}{2 \omega_{1}}\left(1-e^{-\omega_{1} t}\right) \operatorname{tr}_{H}\left(P_{n} \circ Q\right)+\int_{0}^{t} e^{-\omega_{1} s}\left\langle v_{s}^{n}, d P_{n} W_{s}\right\rangle_{H}
\end{aligned}
$$

and thus

$$
\begin{aligned}
& E\left(\sup _{0 \leq t \leq T}\left\|v_{t}^{n}\right\|_{H}^{2}+2 \omega_{0} \int_{0}^{T}\left\|v_{s}^{n}\right\|_{V}^{2} d s\right) \\
& \leq e^{\omega_{1} T} E\left(\left\|P_{n} \xi_{0}\right\|_{H}^{2}\right)+\frac{e^{\omega_{1} T}}{\omega_{1}} \operatorname{tr}_{H}\left(P_{n} \circ Q\right) \\
& \quad+e^{\omega_{1} T} E\left(\sup _{0 \leq t \leq T}\left|\int_{0}^{t} e^{-\omega_{1} s}\left\langle v_{s}^{n}, d P_{n} W_{s}\right\rangle\right|\right) .
\end{aligned}
$$


Since the stochastic integral $\int_{0}^{t} e^{-\omega_{1} s}\left\langle v_{s}^{n}, d P_{n} W_{s}\right\rangle, t \geq 0$, is a continuous $\left(\mathcal{F}_{t}\right)_{t \geq 0^{-}}$ martingale, Doob's maximal inequality implies that

$$
\begin{aligned}
E\left(\sup _{0 \leq t \leq T}\left|\int_{0}^{t} e^{-\omega_{1} s}\left\langle v_{s}^{n}, d P_{n} W_{s}\right\rangle\right|\right) & \leq 2 E\left(\left(\int_{0}^{T} e^{-\omega_{1} s}\left\langle v_{s}^{n}, d P_{n} W_{s}\right\rangle\right)^{2}\right)^{\frac{1}{2}} \\
& =2 E\left(\int_{0}^{T} e^{-2 \omega_{1} s}\left\|\sqrt{Q} v_{s}^{n}\right\|_{H}^{2} d s\right)^{\frac{1}{2}} \\
& \leq 2\|\sqrt{Q}\|_{\mathrm{op}} \sqrt{T} \cdot E\left(\sup _{0 \leq t \leq T}\left\|v_{t}^{n}\right\|_{H}^{2}\right)^{\frac{1}{2}} \\
& \leq \frac{1}{2} e^{-\omega_{1} T} E\left(\sup _{0 \leq t \leq T}\left\|v_{t}^{n}\right\|_{H}^{2}\right)+2 e^{\omega_{1} T}\|\sqrt{Q}\|_{\mathrm{op}}^{2} T .
\end{aligned}
$$

Here, $\|\sqrt{Q}\|_{\text {op }}$ denotes the usual operator norm of $\sqrt{Q}$ on $H$. Inserting the last inequality into (3.9) we obtain the energy estimate

$$
\begin{aligned}
& E\left(\sup _{0 \leq t \leq T}\left\|v_{t}^{n}\right\|_{H}^{2}+4 \omega_{0} \int_{0}^{T}\left\|v_{s}^{n}\right\|_{V}^{2} d s\right) \\
& \leq 2 e^{\omega_{1} T} E\left(\left\|P_{n} \xi_{0}\right\|_{H}^{2}\right)+2 \frac{e^{\omega_{1} T}}{\omega_{1}} \operatorname{tr}_{H}\left(P_{n} \circ Q\right)+4 e^{2 \omega_{1} T} T\|\sqrt{Q}\|_{\mathrm{op}}^{2} \\
& \leq 2 e^{\omega_{1} T} E\left(\left\|\xi_{0}\right\|_{H}^{2}\right)+2 \frac{e^{\omega_{1} T}}{\omega_{1}} \operatorname{tr}_{H}(Q)+4 e^{2 \omega_{1} T} T\|\sqrt{Q}\|_{\mathrm{op}}^{2}=: M
\end{aligned}
$$

uniformly in $n$.

Step 3: Tightness of $P^{n}:=P \circ\left(v .^{n}\right)^{-1}$

We next show that $P^{n}, n \geq 1$, is tight on the space $L^{2}([0, T] ; H)$. By Prohorov's theorem, it is sufficient to show that

$$
\sup _{n} E\left(\left\|v \cdot{ }^{n}\right\|_{W^{\beta, 2}\left([0, T], D\left(\left(-A_{S}\right)^{-\frac{\alpha}{2}}\right)\right)}\right)<\infty
$$

for some $\alpha>3$ and $\beta \in\left(0, \frac{1}{2}\right)$ since the embedding

$$
L^{2}([0, T] ; V) \cap W^{\beta, 2}\left([0, T] ; D\left(A_{S}^{-\frac{\alpha}{2}}\right)\right) \hookrightarrow L^{2}([0, T] ; H)
$$

is compact (see [10, Theorem 2.1]). Here, for a given Banach space $X$, the space $W^{\beta, p}([0, T] ; X)$ denotes the Slobodeckii space defined to be the space consisting of all $u \in L^{p}([0, T] ; X)$ satisfying

$$
\|u\|_{W^{\beta, p}([0, T] ; X)}^{p}:=\int_{0}^{T} \int_{0}^{T} \frac{|u(s)-u(t)|_{X}^{p}}{|s-t|^{1+\beta p}} d s d t<\infty,
$$


and the space $L^{2}([0, T] ; V) \cap W^{\beta, 2}\left([0, T] ; D\left(A_{S}^{-\frac{\alpha}{2}}\right)\right)$ is endowed with the natural norm

$$
\|u\|_{L^{2}([0, T] ; V)}+\|u\|_{W^{\beta, p}\left([0, T] ; D\left(\left(-A_{S}\right)^{-\frac{\alpha}{2}}\right)\right.} \cdot
$$

Note that [10, Theorem 2.1] can be applied, since the embedding $V \hookrightarrow H$ is compact. We postpone the proof of (3.10) to Lemma 3.4.

Since $P^{n}$ is tight, actually tight on $L^{2}([0, T] ; H) \cap C\left([0, T] ; D\left(\left(-A_{S}\right)^{-\frac{\alpha}{2}}\right)\right)$ for all $\alpha>3$, we may choose a subsequence, again denoted by $\left(P^{n}\right)$, converging weakly to some probability measure $P$ on $L^{2}([0, T] ; H) \cap C\left([0, T] ; D\left(\left(-A_{S}\right)^{-\frac{\alpha}{2}}\right)\right)$.

Step 4: The limit $n \rightarrow \infty$

This part of the proof is completely analogous to Step 3 of the proof of [10, Theorem 3.1]. We hence only give a brief sketch of it.

Fix $\alpha>3$. Then Skorohod's embedding theorem (see [19]) implies that there exists a stochastic basis $\left(\tilde{\Omega}, \tilde{\mathcal{F}},\left(\tilde{\mathcal{F}}_{t}\right)_{t \geq 0}, \tilde{P}\right)$ and previsible processes $\tilde{v}^{n}, v$. $\in$ $L^{2}([0, T] ; H) \cap C\left([0, T], D\left(A_{S}^{-\frac{\alpha}{2}}\right)\right)$ with

$$
P \circ\left(v^{n}\right)^{-1}=\tilde{P} \circ\left(\tilde{v}^{n}\right)^{-1}, \quad n \in \mathbb{N}
$$

and $\tilde{v}^{n} \rightarrow \tilde{v}$. $\tilde{P}-a . s$ in $L^{2}([0, T] ; H) \cap C\left([0, T], D\left(\left(-A_{S}\right)^{-\frac{\alpha}{2}}\right)\right)$. The uniform energy estimate (3.7) implies by Fatou's lemma, the estimate

$$
\tilde{E}\left(\sup _{0 \leq t \leq T}\left\|\tilde{v}_{t}\right\|_{H}^{2}+\int_{0}^{T}\left\|\tilde{v}_{t}\right\|_{V}^{2} d t\right)<\infty .
$$

Hence, $\tilde{v} . \in L^{2}([0, T] ; V)$. In order to identify the limiting process $\tilde{v}$. as a martingale solution of (1.5) note that for all $n$

$$
\tilde{M}_{t}^{n}:=\tilde{v}_{t}^{n}-P_{n} \xi_{0}-\int_{0}^{t} P_{n}\left(\nu A_{S}+B\right) \tilde{v}_{s}^{n} d s-\int_{0}^{t} P_{n} F_{n}\left(\tilde{v}_{s}^{n}, \tilde{v}_{s}^{n}\right) d s,
$$

$t \in[0, T]$, is a continuous square-integrable martingale w.r.t $\tilde{\mathcal{G}}_{t}^{n}:=\sigma\left(\tilde{v}_{t}^{n}: s \in\right.$ $[0, t])$ converging $\tilde{P}$-a.s. to

$$
\tilde{M}_{t}:=\tilde{v}_{t}-\xi_{0}-\int_{0}^{t}\left(\nu A_{S}+B\right) \tilde{v}_{s} d s-\int_{0}^{t}\left(\tilde{v}_{s} \cdot \nabla \tilde{v}_{s}\right) d s
$$

for all $t \in[0, T]$ in $D\left(A_{S}^{-\frac{\alpha}{2}}\right)$ for all $\alpha>3$.

This implies that $\tilde{M}_{t}, t \in[0, T]$, is a martingale w.r.t. the filtration $\tilde{\mathcal{G}}_{t}:=$ $\sigma\left(\tilde{v}_{t}: s \in[0, t]\right)$. The martingale representation theorem implies that there exists a $Q$-Wiener process $\left(\tilde{W}_{t}\right)_{t \geq 0}$ on $(\tilde{\Omega}, \tilde{\mathcal{F}}, \tilde{P})$ such that

$$
\left\langle\tilde{M}_{t}, u\right\rangle=\left\langle\tilde{W}_{t}, u\right\rangle \quad \tilde{P}-a . s ., u \in V .
$$

The proof is complete. 
We finally prove the tightness of $P^{n}$.

Lemma 3.4. Let $\alpha>3, \beta \in\left(0, \frac{1}{2}\right)$. Then

$$
\sup _{n} E\left(\left\|v^{n}\right\|_{W^{\beta, 2}\left([0, T] ; D\left(\left(-A_{S}\right)^{-\frac{\alpha}{2}}\right)\right)}\right)<\infty \text {. }
$$

Proof. We decompose the unique strong solution $v_{t}^{n}$ of (3.5) as

$$
\begin{aligned}
v_{t}^{n} & =P_{n} \xi_{0}+\int_{0}^{t} P_{n}\left(v A_{S}+B\right) v_{s}^{n} d s+\int_{0}^{t} P_{n} F_{n}\left(v_{s}^{n}, v_{s}^{n}\right) d s+P_{n} W_{t} \\
& =: I_{1}+I_{2}(t)+I_{3}(t)+I_{4}(t) .
\end{aligned}
$$

It is then sufficient to show the desired estimate for each term separately. Clearly, for $I_{1}$, we obtain

$$
\left\|I_{1}\right\|_{W^{\beta, 2}\left([0, T] ; D\left(\left(-A_{S}\right)^{-\frac{\alpha}{2}}\right)\right)}=\sqrt{T}\left\|P_{n} \xi_{0}\right\|_{D\left(\left(-A_{S}\right)^{-\frac{\alpha}{2}}\right)} \leq C \cdot \sqrt{T} \cdot\left\|\xi_{0}\right\|_{H}
$$

for some uniform constant $C>0$, so that

$$
\sup _{n} E\left(\left\|I_{1}\right\|_{W^{\beta, 2}\left([0, T] ; D\left(\left(-A_{S}\right)^{-\frac{\alpha}{2}}\right)\right)}\right)<\infty .
$$

By Lemma 2.1 in [10],

$$
\left\|P_{n} W .\right\|_{W^{\beta, 2}\left([0, T] ; D\left(A_{S}^{-\frac{\alpha}{2}}\right)\right)}^{2} \leq C(\beta, 2) \cdot T \cdot \operatorname{tr}_{H}\left(\sqrt{Q} \circ P_{n} \circ \sqrt{Q}\right)
$$

for some uniform constant $C(\beta, 2)$. Thus

$$
\sup _{n} E\left(\left\|I_{4}(\cdot)\right\|_{W^{\beta, 2}\left([0, T] ; D\left(A_{S}^{-\frac{\alpha}{2}}\right)\right)}\right)<\infty .
$$

Concerning $I_{2}$, note that $\dot{I}_{2}(t)=P_{n}\left(v A_{S}+B\right) v_{t}^{n}$, and thus

$$
\left\|\dot{I}_{2}(t)\right\|_{V^{\prime}}=\left\|P_{n}\left(v A_{S}+B\right) v_{t}^{n}\right\|_{V^{\prime}} \leq C\left\|v_{t}^{n}\right\|_{V}
$$

for some universal constant $C$, because

$$
\begin{aligned}
\left\langle P_{n}\left(v A_{S}+B\right) v_{t}^{n}, u\right\rangle_{H} & =v\left\langle A_{S} v_{t}^{n}, P_{n} u\right\rangle_{H}+\left\langle B v_{t}^{n}, P_{n} u\right\rangle_{H} \\
& =v\left\langle A_{S} v_{t}^{n}, u\right\rangle_{H}+\left\langle B v_{t}^{n}, P_{n} u\right\rangle_{H} \\
& \leq v\left\|v_{t}^{n}\right\|_{V} \cdot\|u\|_{V}+\left\|B v_{t}^{n}\right\|_{H}\left\|P_{n} u\right\|_{H} \\
& \leq C\left\|v_{t}^{n}\right\|_{V} \cdot\|u\|_{V} .
\end{aligned}
$$

Here we used assumption (A.1) and the fact that $\left\|P_{n} u\right\|_{H} \leq\|u\|_{H} \leq C\|u\|_{V}$ holds for some uniform constant $C>0$. Consequently,

$$
\sup _{n} E\left(\int_{0}^{T}\left\|\dot{I}_{2}(t)\right\|_{V^{\prime}}^{2} d t\right) \leq C \sup _{n} E\left(\int_{0}^{T}\left\|v_{t}^{n}\right\|_{V}^{2} d t\right)<\infty,
$$


by using the energy estimate (3.7). Since $\left\|I_{2}(\cdot)\right\|_{W^{\beta, 2}\left([0, T] ; D\left(\left(-A_{S}\right)^{-\frac{\alpha}{2}}\right)\right)}^{2}$ can obviously be estimated from above by $\left\|I_{2}(\cdot)\right\|_{W^{1,2}\left([0, T] ; V^{\prime}\right)}^{2}$ up to some uniform constant, we obtain the desired uniform estimate

$$
\sup _{n} E\left(\left\|I_{2}(\cdot)\right\|_{W^{\beta, 2}\left([0, T] ; D\left(A_{S}^{-\frac{\alpha}{2}}\right)\right)}\right)<\infty .
$$

Finally, note that

$$
\begin{aligned}
\left\langle P_{n} F_{n}\left(v_{t}^{n}, v_{t}^{n}\right), u\right\rangle_{H} & =\left\langle\Theta_{n}\left(\left\|v_{t}^{n}\right\|_{H}\right) v_{t}^{n} \cdot \nabla v_{t}^{n}, P_{n} u\right\rangle_{H} \\
& \leq \int_{\Omega_{b}}\left|v_{t}^{n} \cdot \nabla v_{t}^{n}\right|_{\mathbb{R}^{3}} d x \cdot\left\|\left|P_{n} u\right|_{\mathbb{R}_{3}}\right\|_{\infty} \\
& \leq C\left\|v_{t}^{n}\right\|_{H} \cdot\left\|v_{t}^{n}\right\|_{V}\|u\|_{D\left(\left(-A_{S}\right)^{\frac{\alpha}{2}}\right)}
\end{aligned}
$$

for some uniform constant, since

$$
\left\|\left|P_{n} u\right|_{\mathbb{R}^{3}}\right\|_{\infty} \leq C\left\|P_{n} u\right\|_{D\left(\left(-A_{S}\right)^{\frac{\alpha}{2}}\right)}
$$

for $\alpha>3$. Hence,

$$
\left\|P_{n} F_{n}\left(v_{t}^{n}, v_{t}^{n}\right)\right\|_{D\left(A_{S}^{-\frac{\alpha}{2}}\right)} \leq C\left\|v_{t}^{n}\right\|_{H}\left\|v_{t}^{n}\right\|_{V}
$$

for some uniform constant. This implies that $I_{3}$ is differentiable with respect to $t$ in $D\left(A_{S}^{-\frac{\alpha}{2}}\right)$ and that

$$
\left\|\dot{I}_{3}(t)\right\|_{D\left(\left(-A_{S}\right)^{\left.-\frac{\alpha}{2}\right)}\right.} \leq C\left\|v_{t}^{n}\right\|_{H}\left\|v_{t}^{n}\right\|_{V}
$$

Thus

$$
\left\|I_{3}\right\|_{W^{1,2}\left([0, T] ; D\left(A_{S}^{-\frac{\alpha}{2}}\right)\right)}^{2} \leq C \sup _{0 \leq t \leq T}\left\|v_{t}^{n}\right\|_{H}^{2} \cdot \int_{0}^{T}\left\|v_{t}^{n}\right\|_{V}^{2} d t,
$$

and

$$
E\left(\left\|I_{3}(\cdot)\right\|_{W^{1,2}\left([0, T] ; D\left(\left(-A_{S}\right)^{\left.\left.-\frac{\alpha}{2}\right)\right)}\right.\right.}\right) \leq \frac{C}{2} E\left(\sup _{0 \leq t \leq T}\left\|v_{t}^{n}\right\|_{H}^{2}+\int_{0}^{T}\left\|v_{t}^{n}\right\|_{V}^{2} d t\right)
$$

is uniformly bounded in $n$ which finally implies the desired estimate for $I_{3}$. Summarizing, the lemma is proven.

We now may apply Theorem 3.3 to the stochastic evolution equation (1.6), with $B$ given by

$$
B v=\omega \Pi\left(e_{3} \times v\right)-\Pi\left(u_{b}^{E} \cdot \nabla v\right)-\Pi\left(v_{3} \partial_{3} u_{b}^{E}\right) .
$$

Since $B$ satisfies assumption (A.1), we obtain from Theorem 3.3 that for any $\mu \in$ $\mathcal{M}_{1}(H)$ satisfying $\int\|x\|_{H}^{2} d \mu(x)<\infty$, there exists a martingale solution $\left(\left(v_{t}\right)_{t \geq 0},\left(W_{t}\right)_{t \geq 0}\right)$ of (1.6) satisfying $\tilde{P}_{0} \circ v_{0}^{-1}=\mu$ and the energy estimate

$$
E\left(\sup _{0 \leq t \leq T}\left\|v_{t}\right\|_{H}^{2}+\int_{0}^{T}\left\|v_{s}\right\|_{V}^{2} d s\right)<\infty .
$$


Defining the stochastic process

$$
u_{t}:=v_{t}+u_{b}^{E}, t \geq 0
$$

it follows that $\left(\left(u_{t}\right)_{t \geq 0},\left(W_{t}\right)_{t \geq 0}\right)$ is a martingale solution of (1.6). Thus, the following corollary holds

Corollary 3.5. Let $\mu \in \mathcal{M}_{1}(H)$ with $\int\|x\|_{H}^{2} d \mu(x)<\infty$. Then there exists a weak martingale solution of the stochastic Navier-Stokes-Coriolis-Ekman equation (1.6) with initial distribution $\mu$.

\section{Stationary martingale solutions and invariant measures}

We recall that a stochastic process $\left(X_{t}\right)_{t \geq 0}$ is called stationary if the distribution of the time-shifted process $\left(X_{s+} \cdot\right)_{t \geq 0}$ is independent of $s$, i.e.,

$$
P \circ\left(X_{s+\cdot}\right)^{-1}=P \circ(X .)^{-1}, \quad s \geq 0 .
$$

Stationarity implies in particular that the distribution of $X_{t}$ is independent of $t$. Hence, $\mu=P \circ X_{0}^{-1}$ is called an invariant probability measure.

A particular example for a stationary process is a Markov process $\left(\left(X_{t}\right)_{t \geq 0}\right.$, $\left.\left(P_{x}\right)_{x \in E}\right)$ on a state space $(E, \mathcal{E})$ having an invariant measure $\mu$ in the sense that for any $\mathcal{E}$-measurable bounded function $F$

$$
\int E_{x}\left(F\left(X_{t}\right)\right) \mu(d x)=\int F(x) \mu(d x), \quad t \geq 0 .
$$

In this case, the distribution of the shifted process $\left(X_{S+t}\right)_{t \geq 0}$ with respect to the probability measure

$$
P_{\mu}(A):=\int P_{x}(A) \mu(d x)
$$

will be independent of $s$.

In the case of the stochastic Navier-Stokes-Coriolis equation we are yet far away from the construction of a full Markov process, however using the same techniques as in Section 3, we are able to construct a stationary martingale solution under additional assumptions on the coefficients. To this end, consider again first the perturbed stochastic Navier-Stokes equation (1.6) for general $B$ satisfying assumption (A.1) with $\omega_{1}<\frac{\pi^{2}}{b^{2}} \omega_{0}$. Then

$$
\left\langle\left(v A_{S}+B\right) u, u\right\rangle_{H} \leq-\tilde{\omega}_{0}\|u\|_{V}^{2}, \quad u \in D\left(A_{S}\right) .
$$

with $\tilde{\omega}_{0}:=\omega_{0}-\frac{b^{2}}{\pi^{2}} \omega_{1}>0$. 
Theorem 4.1. There exists a stationary martingale solution $\left(\left(v_{t}\right)_{t \geq 0},\left(W_{t}\right)_{t \geq 0}\right)$ of (1.6) satisfying

$$
E\left(\sup _{0 \leq t \leq T}\left\|v_{t}\right\|_{H}^{2}+\int_{0}^{T}\left\|v_{t}\right\|_{V}^{2} d t\right)<\infty, \quad T>0 .
$$

Moreover, the invariant distribution $\mu=P \circ v_{t}^{-1}$ satisfies the moment estimates

$$
\int e^{\varepsilon\|x\|_{H}^{2}} \mu(d x)<\infty \quad \text { for } \varepsilon<\varepsilon_{0}:=\frac{\tilde{\omega}_{0}}{\|Q\|_{\text {op }}} \frac{\pi^{2}}{b^{2}}
$$

and

$$
\int\|x\|_{V}^{2} \mu(d x)<\infty
$$

Proof. Similar to the proof of Theorem 3.3, we consider the finite dimensional Galerkin approximation

$$
d v_{t}^{n}=\left[P_{n}\left(v A_{S}+B\right) v_{t}^{n}+P_{n} F_{n}\left(v_{t}^{n}, v_{t}^{n}\right)\right] d t+d P_{n} W_{t} .
$$

Equation (4.5) has a unique strong solution $v^{n}\left(t, v_{0}\right)$ for any initial condition $v_{0} \in$ $P_{n} H$. Moreover, the associated transition semigroup

$$
P_{t}^{n} F\left(v_{0}\right):=E\left(F\left(v^{n}\left(t, v_{0}\right)\right)\right) \quad t \geq 0,
$$

has the Feller property, i.e. $P_{t}^{n}\left(C_{b}\left(P_{n} H\right)\right) \subset C_{b}\left(P_{n} H\right)$, where $C_{b}\left(P_{n} H\right)$ denotes the space of bounded continuous functions on $P_{n} H$.

Ito's formula, applied to $\frac{1}{2}\left\|v^{n}\left(t, v_{0}\right)\right\|_{H}^{2}$, yields the estimate

$$
\begin{aligned}
\frac{1}{2}\left\|v^{n}\left(t, v_{0}\right)\right\|_{H}^{2} \leq & \frac{1}{2}\left\|v_{0}\right\|_{H}^{2}+\int_{0}^{t}\left\langle v_{s}^{n}, d P_{n} W_{s}\right\rangle_{H} \\
& -\tilde{\omega}_{0} \int_{0}^{t}\left\|v^{n}\left(s, v_{0}\right)\right\|_{V}^{2} d s+\frac{t}{2} \cdot \operatorname{tr}_{H}\left(P_{n} \circ Q\right) .
\end{aligned}
$$

In particular, taking expectations we conclude that

$$
E\left(\frac{1}{t} \int_{0}^{t}\left\|v^{n}\left(s, v_{0}\right)\right\|_{V}^{2} d s\right) \leq \frac{1}{2 t \tilde{\omega}_{0}}\left\|v_{0}\right\|_{H}^{2}+\frac{1}{2 \tilde{\omega}_{0}} \operatorname{tr}_{H}\left(P_{n} \circ Q\right),
$$

which implies that the family $\mu_{n}(t, A):=\frac{1}{t} \int_{0}^{t} P\left(v^{n}\left(s, v_{0}\right) \in A\right) d s, A \in \mathcal{B}\left(P_{n} H\right)$, $t \geq 0$, of the mean occupation time measures is tight on $P_{n} H$. The KrylovBogoliubov Theorem (see [7]) implies the existence of an invariant probability measure $\mu_{n}$ on $P_{n} H$ for $\left(P_{t}^{n}\right)$, i.e.,

$$
\int_{P_{n} H} P_{t}^{n} F d \mu_{n}=\int_{P_{n} H} F d \mu_{n}
$$


satisfying the moment estimate

$$
\int_{P_{n} H}\|v\|_{V}^{2} \mu_{n}(d v) \leq \frac{1}{2 \tilde{\omega}_{0}} \operatorname{tr}_{H}\left(P_{n} \circ Q\right) \leq \frac{1}{2 \tilde{\omega}_{0}} \operatorname{tr}_{H}(Q)
$$

uniformly in $n$. Moreover, we construct in the usual way a stationary martingale solution $\left(\left(v_{t}^{n}\right)_{t \geq 0},\left(W_{t}\right)_{t \geq 0}\right)$ with distribution $\mu_{n}$. In Lemma 4.2 below we will prove that for $\varepsilon<\varepsilon_{0}=\frac{\tilde{\omega}_{0}}{\|Q\|_{\mathrm{op}}} \frac{\pi^{2}}{b^{2}}, \mu_{n}$ also satisfies the exponential moment estimate

$$
\int_{P_{n} H} e^{\varepsilon\|v\|_{H}^{2}} \mu_{n}(d v) \leq C_{1}(\varepsilon)
$$

for some finite constant $C_{1}(\varepsilon)$ independent of $n$.

We next show that the family $P_{n}$ of distributions of $\left(v_{t}^{n}\right)_{t \leq 0}$ is tight on $L_{\text {loc }}^{2}([0, T] ; H) \cap C\left([0, T] ; D\left(A_{S}^{-\frac{\alpha}{2}}\right)\right)$ for any $\alpha>3$ and any finite $T$. To this end, it suffices to recall from the proof of Theorem 3.3 that for all $T>0$

$$
\sup _{n} E\left(\left\|v^{n}\left(\cdot, v_{0}\right)\right\|_{W^{\beta, 2}\left([0, T] ; D\left(\left(-A_{S}\right)^{-\frac{\alpha}{2}}\right)\right)}\right)<\infty
$$

for any $\alpha>3$ and $\beta \in\left(0, \frac{1}{2}\right)$, see (3.10). Now, similarly to Step 4 in the proof of Theorem 3.3, we find a stochastic basis $\left(\tilde{\Omega}, \tilde{\mathcal{F}},\left(\tilde{\mathcal{F}}_{t}\right)_{t \geq 0}, \tilde{P}\right)$ and previsible processes $\tilde{v}_{.}^{n}, \tilde{v}$. such that $\tilde{P} \circ\left(\tilde{v}^{n}\right)^{-1}$ coincides with the distribution of $v_{.}^{n}$. In particular, $\tilde{v}^{n}$. is stationary for all $n$, hence $\tilde{v}_{t}=\lim _{n \rightarrow \infty} \tilde{v}_{t}^{n}$ is stationary too. Similar to the proof of Theorem 3.3 we can show that there exists a $Q$-Wiener process $\left(\tilde{W}_{t}\right)_{t \geq 0}$ on $(\tilde{\Omega}, \tilde{\mathcal{F}}, \tilde{P})$ such that

$$
\left\langle\tilde{W}_{t}, u\right\rangle={ }_{V^{\prime}}\left\langle\tilde{v}_{t}-\tilde{v}_{0}-\int_{0}^{t}\left(v A_{S}+B\right) \tilde{v}_{s} d s-\int_{0}^{t} \Pi\left(\tilde{v}_{s} \cdot \nabla \tilde{v}_{s}\right) d s, u\right\rangle_{V} \quad \tilde{P}-a . s ., u \in V .
$$

which implies the assertion of the theorem.

It remains to prove the exponential moment estimate (4.8).

Lemma 4.2. Let $t_{k} \uparrow \infty$ and $\mu_{n}$ be an accumulation point of the mean occupation time measures $\frac{1}{t_{k}} \int_{0}^{t_{k}} P\left(v^{n}\left(s, v_{0}\right) \in A\right) d s, A \in \mathcal{B}\left(P_{n} H\right), k \geq 1$, for some $v_{0} \in$ $P_{n} H$. Then, for $\varepsilon<\varepsilon_{0}=\frac{\tilde{\omega}_{0}}{\|Q\|_{\mathrm{op}}} \frac{\pi^{2}}{b^{2}}$, there exists a finite constant $C_{1}(\varepsilon)$, independent of $n$, such that

$$
\int_{P_{n} H} e^{\varepsilon\|v\|_{H}^{2}} d \mu_{n}(v) \leq C_{1}(\varepsilon) .
$$

Proof. Since for any deterministic initial condition $v_{0},\left\|v_{0}\right\|_{H} \in \mathcal{L}^{m}(P)$ for all $m$, it follows that

$$
E\left(\sup _{0 \leq t \leq T}\left\|v^{n}\left(t, v_{0}\right)\right\|_{H}^{2 m}\right)<\infty \quad \text { for all } T .
$$


Ito's formula implies for all $m \geq 1$

$$
\begin{aligned}
\left\|v^{n}\left(t, v_{0}\right)\right\|_{H}^{2 m} \leq & \left\|v_{0}\right\|_{H}^{2 m} \\
& +m \int_{0}^{t}\left\|v^{n}\left(s, v_{0}\right)\right\|_{H}^{2(m-1)}\left(\operatorname{tr}_{H}\left(P_{n} \circ Q\right)-2 \tilde{\omega}_{0}\left\|v^{n}\left(s, v_{0}\right)\right\|_{V}^{2}\right) d s \\
& +2 m(m-1) \int_{0}^{t}\left\|v^{n}\left(s, v_{0}\right)\right\|_{H}^{2(m-2)}\left\langle Q v^{n}\left(s, v_{0}\right), v^{n}\left(s, v_{0}\right)\right\rangle_{H} d s \\
& +2 m \int_{0}^{t}\left\|v^{n}\left(s, v_{0}\right)\right\|_{H}^{2(m-1)}\left\langle v^{n}\left(s, v_{0}\right), d P_{n} W_{s}\right\rangle_{H} .
\end{aligned}
$$

Since $\left\langle Q v^{n}\left(s, v_{0}\right), v^{n}\left(s, v_{0}\right)\right\rangle_{H} \leq\|Q\|_{\text {op }}\left\|v^{n}\left(s, v_{0}\right)\right\|_{H}^{2}$ we obtain that

$$
\begin{aligned}
& \frac{1}{t} \int_{0}^{t}\left\|v^{n}\left(s, v_{0}\right)\right\|_{V}^{2}\left\|v^{n}\left(s, v_{0}\right)\right\|_{H}^{2(m-1)} d s \\
& \leq \frac{1}{t} \frac{1}{2 \tilde{\omega}_{0} m}\left\|v_{0}\right\|_{H}^{2 m} \\
& \quad+\frac{1}{t} \int_{0}^{t} \frac{\operatorname{tr}\left(P_{n} \circ Q\right)+2(m-1)\|Q\|_{\mathrm{op}}}{2 \tilde{\omega}_{0}} \cdot\left\|v^{n}\left(s, v_{0}\right)\right\|_{H}^{2(m-1)} d s \\
& \quad+\frac{1}{t} \int_{0}^{t} \frac{1}{\tilde{\omega}_{0}}\left\|v^{n}\left(s, v_{0}\right)\right\|_{H}^{2(m-1)}\left\langle v^{n}\left(s, v_{0}\right), d P_{n} W_{s}\right\rangle_{H} .
\end{aligned}
$$

Taking expectations we conclude that for $m \in \mathbb{N}$

$$
\begin{aligned}
& E\left(\frac{1}{t} \int_{0}^{t}\left\|v^{n}\left(s, v_{0}\right)\right\|_{H}^{2(m-1)}\left\|v^{n}\left(s, v_{0}\right)\right\|_{V}^{2} d s\right) \\
& \leq \frac{1}{2} \frac{1}{t \tilde{\omega}_{0} m}\left\|v_{0}\right\|_{H}^{2 m}+\frac{\operatorname{tr}\left(P_{n} \circ Q\right)+2(m-1)\|Q\|_{\mathrm{op}}}{2 \tilde{\omega}_{0}} \cdot E\left(\frac{1}{t} \int_{0}^{t}\left\|v^{n}\left(s, v_{0}\right)\right\|_{H}^{2(m-1)} d s\right) .
\end{aligned}
$$

It is now standard to conclude that (4.9) implies for the limiting measure $\mu_{n}$ first that

$$
\int\|v\|_{H}^{2 m} d \mu_{n}(v) \leq C(m), \quad m \in \mathbb{N}
$$

for some finite constant $C(m)$ independent of $n$ and then, by taking the limit $t \rightarrow \infty$ in (4.9),

$$
\begin{aligned}
& \int\|v\|_{V}^{2}\|v\|_{H}^{2(m-1)} d \mu_{n}(v) \\
& \leq \frac{\operatorname{tr}\left(P_{n} \circ Q\right)+2(m-1)\|Q\|_{\text {op }}}{2 \tilde{\omega}_{0}} \int\|v\|_{H}^{2(m-1)} d \mu_{n}(v), \quad m \in \mathbb{N} .
\end{aligned}
$$

For $\varepsilon<\varepsilon^{\prime}<\varepsilon_{0}$ let $M_{\varepsilon} \in \mathbb{N}$ be such that

$$
\varepsilon \operatorname{tr}_{H}(Q) \leq\left(\varepsilon^{\prime}-\varepsilon\right) 2(m-1)\|Q\|_{\text {op }} \quad \text { for all } m \geq M_{\varepsilon} \text {. }
$$


Since $\|v\|_{H}^{2} \leq \frac{b^{2}}{\pi^{2}}\|v\|_{V}^{2}$, we obtain for $M \in \mathbb{N}, M \geq M_{\varepsilon} \in \mathbb{N}$, that

$$
\sum_{k=M_{\varepsilon}}^{M} \frac{\varepsilon^{k}}{k !} \int\|v\|_{H}^{2 k} d \mu_{n}(v) \leq \varepsilon^{\prime} \frac{b^{2}}{\pi^{2}} \frac{\|Q\|_{\mathrm{op}}}{\tilde{\omega}_{0}} \sum_{k=M_{\varepsilon}-1}^{M-1} \frac{\varepsilon^{k}}{k !} \int\|v\|_{H}^{2 k} d \mu_{n}(v),
$$

and thus

$$
\sum_{k=0}^{M} \frac{\varepsilon^{k}}{k !} \int\|v\|_{H}^{2 k} d \mu_{n}(v) \leq \frac{1}{1-\varepsilon^{\prime} / \varepsilon_{0}} \sum_{k=0}^{M_{\varepsilon}-1} \frac{\varepsilon^{k}}{k !} \int\|v\|_{H}^{2 k} d \mu_{n}(v) .
$$

The right hand side above does not depend on $M$, so that we may take the limit $M \rightarrow \infty$ to obatin the exponential moment estimate

$$
\int e^{\varepsilon\|v\|_{H}^{2}} d \mu_{n}(v) \leq \frac{\varepsilon_{0}}{\varepsilon_{0}-\varepsilon^{\prime}} \sum_{k=0}^{M_{\varepsilon}-1} \frac{\varepsilon^{k}}{k !} C(k)=: C_{1}(\varepsilon) .
$$

for some finite constant $C_{1}(\varepsilon)$ independent of $n$.

Finally, consider again the stochastic evolution equation (1.6) with

$$
B v:=-\omega \Pi\left(e_{3} \times v\right)-\Pi\left(u_{b}^{E} \cdot \nabla v\right)-\Pi\left(v_{3} \cdot \partial_{3} u_{b}^{E}\right) .
$$

If

$$
\frac{\tilde{u}_{b}}{v}\left(\delta-b e^{-\frac{b}{\delta}}-\delta e^{-\frac{b}{\delta}}\right)<\frac{1}{\sqrt{2}},
$$

then $B$ satisfies assumption (A.1) with $\omega_{1}<\frac{\pi^{2}}{b^{2}} \omega_{0}$, so that

$$
\left\langle\left(v A_{S}+B\right) u, u\right\rangle_{H} \leq-\tilde{\omega}_{0}\|u\|_{V}^{2}
$$

with $\tilde{\omega}_{0}=\omega_{0}-\frac{b^{2}}{\pi^{2}} \omega_{1}$. Hence, there exists a stationary martingale solution $\left(\left(v_{t}\right)_{t \geq 0},\left(W_{t}\right)_{t \geq 0}\right)$ of (1.6) satisfying (4.2) and its distribution $P \circ v_{t}^{-1}$ on $H$ satisfies the moment estimates (4.3) and (4.4).

Considering the stochastic process

$$
u_{t}=v_{t}+u_{b}^{E}, t \geq 0,
$$

we verify that $\left(\left(u_{t}\right)_{t \geq 0},\left(W_{t}\right)_{t \geq 0}\right)$ is a stationary martingale solution of (1.5), which proves the following corollary.

Corollary 4.3. Assume that equation (4.11) holds. Then there exists a stationary martingale solution $\left(\left(u_{t}\right)_{t \geq 0},\left(W_{t}\right)_{t \geq 0}\right)$ of the stochastic Navier-Stokes-CoriolisEkman equation (1.6). Its invariant distribution $\mu=P \circ u_{t}^{-1}$ satisfies the moment estimates (4.3) and (4.4). 


\section{References}

[1] T. ABE and Y. SHIBATA, On a resolvent estimate of the Stokes equation on an infinite layer, J. Math. Soc. Japan 55 (2003), 469-497.

[2] H. AbELS and M. WIEGNER, Resolvent estimates for the Stokes equation on an infinite layer, Differential Integral Equations 18 (2005), 1081-1110.

[3] A. Babin, A. Mahalov and B. NicolaenKo, 3D Navier-Stokes and Euler equations with initial data characterized by uniformly large vorticity, Indiana Univ. Math. J. 50 (2001), $1-35$.

[4] A. Bensoussan and R. Temam, Équations stochastiques du type Navier-Stokes, J. Func. Anal. 13 (1973), 195-222.

[5] J. Bricmont, A. Kupiainen and R. Lefevere, Probabilistic estimates for the twodimensionl stochatic Navier-Stokes equations, J. Statist. Phys. 100 (2000), 743-756.

[6] G. DA PRATO and J. ZABCZYK, "Stochastic Equations in Infinite Dimensions", Cambridge University Press, 1992.

[7] G. DA PRATo and J. ZAвCZYк, "Ergodicity for Infinite Dimensional Systems", Lectures Note Series of the LMS, Vol. 229, Cambridge University Press, 1996.

[8] B. Desjardins, E. Dormy and E. Grenier, Stability of mixed Ekman-Hartmann boundary layers, Nonlinearity 12 (1999), 181-199.

[9] V. W. EKMAn, On the influence of the earth's rotation on ocean currents, Arkiv Matem. Astr. Fysik (Stockholm) 11 (1905), 1-52.

[10] F. FlANDOLI and D. GATAREK, Martingale and stationary solutions for stochastic NavierStokes equations, Probab. Theory Rel. Fields 102 (1995), 367-391.

[11] G. GALDI, "An Introduction to the Mathematical Theory of the Navier-Stokes Equations", Springer, Berlin, 1994.

[12] TH. GALLAY and V. RousSiER-MichON, Global existence and long-time asymptotics for rotating fluids in a 3D-layer, J. Math. Anal. Appl. 360 (2009), 14-34.

[13] Y. Giga, K. InUi, A. Mahalov, S. Matsui and J. SAal, Rotating Navier-Stokes equations in $\mathbb{R}_{+}^{3}$ with initial data nondecreasing at infinity: the Ekman boundary layer problem, Arch. Ration. Mech. Anal. 186 (2007), 177-224.

[14] Y. GIGA, K. INUI, A. MAHALOV and J. SAAL, Uniform global solvability of the rotating Navier-Stokes equations for nondecaying initial data, Indiana Univ. Math. J. 57 (2008), 2775-2792.

[15] H. HeCK, H. KIm and H. Kozono, Stability of plane Couette flows with respect to small periodic perturbations, Nonlinear Anal. 71 (2009), 3739-3758.

[16] M. Hess, "Analysis of the Navier-Stokes Equations for Geophysical Boundary Layers", PhD-Thesis, TU Darmstadt, 2009.

[17] M. Hess, M. Hieber, A. Mahalov and J. SAAl, Nonlinear stability of the Ekman spiral, Bull. London Math. Soc. 42 (2010), 691-706.

[18] M. Hieber and Y. ShibATA, The Fujita-Kato approach to the equations of Navier-Stokes in the rotational setting, Math. Z. 265 (2010), 481-491.

[19] N. IKEDA and S. WATANABE, "Stochastic Differential Equations and Diffusion Processes", North Holland, Amsterdam 1981.

[20] N. MASMOUDI, Ekman layers for rotating fluids: the case of general data, Comm. Pure Appl. Math. 53 (2000), 432-483.

[21] N. V. KRYLOV, "Introduction to the Theory of Diffusion Processes", AMS Mathematical Monographs, Vol. 142, AMS, Providence 1995.

[22] C. ODASSO, Spatial smoothness of the stationary solutions ot the 3D Navier-Stokes equations, Electron. J. Probab. 11 (2006), 686-699.

[23] F. Rousset, Stability of large Ekman boundary layers in rotating fluids, Arch. Ration. Mech. Anal. 172 (2004), 213-245.

[24] A. SHIRIKYAn, Analyticity of solutions of randomly perturbed two-dimensional NavierStokes equations, Russian Math. Surveys 57 (2002), 785-799. 
[25] R. Temam, "Navier-Stokes Equations: Theory and Numerical Analysis", North-Holland, Amsterdam, 1984.

[26] M. VishiK and A. Fursikov, "Mathematical Problems of Statistical Hydromechanics", Kluwer, Dordrecht, 1988.

Fachbereich Mathematik

Technische Universität Darmstadt

Schloßgartenstraße 7

64289 Darmstadt, Germany

hieber@mathematik.tu-darmstadt.de

stannat@mathematik.tu-darmstadt.de 